\title{
MicroRNA-16-5p inhibits the Proliferation and Invasion of Glioma Cells by the Activation of p53/BAX Signal Pathway
}

\author{
SHAO CAI HAO, ZHENG LIU, JIN FENG, HUI MA* AND HE CHUN XIA* \\ Department of Neurosurgery, The General Hospital of Ningxia Medical University, Ningxia Key Laboratory of Cerebrocranial \\ Diseases, Incubation Base of National Key Laboratory, Ningxia Medical University 804 Shengli Street, Yinchuan, Ningxia \\ 750004, China
}

Hao et al.: miRNA-16-5p inhibits the Glioma Cells by the Activation of p53/BAX Signal Pathway

\begin{abstract}
Glioma was one of the most common malignant brain tumors. The effect of routine clinical treatment of glioma was not ideal, and the molecular mechanism of the occurrence and development of the disease was still unclear. In this paper, the mechanism of MicroRNA-16-5p in the occurrence and development of glioma was studied by the cell experiment in vitro. Through the analysis of the The Cancer Genome Atlas database and clinical tissue samples, the expression of MicroRNA-16-5p in glioma was detected. The inhibitory effect of MicroRNA-16-5p on the proliferation, invasion, cell cycle and apoptosis of glioma cells was evaluated by the cell proliferation, invasion and flow cytometry. The relationship between the expression of p53, Bcl-2 Associated X-protein and MicroRNA-16-5p was studied by dual-luciferase gene assay, Quantitative Reverse transcription- polymerase chain reaction, and Western blotting. Compared with the normal tissues, the expression of MicroRNA-16-5precursor was low in glioma tissues, which was consistent with that in the Uppsala87 and Uppsala251 cell lines. The over expression of MicroRNA-16-5p can inhibit the growth and invasion of the glioma cell line, promote the apoptosis and delay the cell cycle. The results of Quantitative Reverse transcription- polymerase chain reaction and Western blotting showed that the target gene of MicroRNA-16-5p was p53, and the over expression of MicroRNA-16-5p could promote the expression of p53 and Bcl-2 Associated X-protein, which was positively correlated. MicroRNA-16-5p was a tumor suppressor in the progression of glioma. We have demonstrated the MicroRNA-873-5p-p53/ Bcl-2 Associated X-protein regulatory pathway in the development of glioma, which provides a theoretical basis for the development of the alternative strategies for the clinical treatment of glioma.
\end{abstract}

Key words: Alzheimer's disease, extended nursing, behavioral and psychological symptoms, cognitive function, ability of daily life activities

Glioma (GM) was the most common primary brain tumor. In the past $40 \mathrm{y}, \mathrm{GM}$ has the highest mortality among all brain malignant tumors ${ }^{[1]}$. There were about 20000 new cases of GM in the United States every $y^{[2]}$. Despite surgery, chemotherapy and/or radiotherapy, the median survival time for the patients with GM was only 12 to $14 \mathrm{mo}^{[3]}$. Therefore, it is necessary to explore the mechanism of the development and progress of GM and to find a more effective treatment.

MicroRNA was a non-coding small molecule RNA with a length of 22-25 nt, which exists in eukaryotes. A large number of studies have shown that the miRNA can regulate not only the biological effects such as proliferation, apoptosis, senescence and differentiation of GM cells $\mathrm{s}^{[4-7]}$, but also autophagy and drug resistance of $\mathrm{GM}^{[8,9]}$. Therefore, it has been proposed as a new target for the anticancer therapy in recent $y^{[7,8]}$. More than $50 \%$ of the miRNA was known to be involved in the human tumorigenesis by directly targeting the oncogenes or tumor suppressor genes ${ }^{[9]}$. Recent studies have shown that the miR-16 can inhibit the proliferation, migration, invasion and other tumor suppressor genes of GM cells, which may be related to the low expression of Sal-like protein 4 (SALL4), zyxin, Cyclin D1 (CCND1), Cyclin E1 (CCNE1), B lymphoma Mo-MLV insertion region 1 homolog (BMI1), Wip1 and other signal proteins ${ }^{[8-12]}$. Therefore, miR-16 can be used as a potential target for the treatment of GM. 
$\mathrm{p} 53^{[13]}$ is the tumor suppressor gene at present. Nearly 5000 studies have been published on the inhibitory role of p53 in cancer progression, including its significance in the GM mechanism. p53 was initially mistaken for p53 oncogene ${ }^{[14]}$ because of the high expression of its mutant Complementary DNA (cDNA) in various cancers. In fact, the inhibition of p53 can activate the occurrence of cancer, which has a significant effect on the cancer cell cycle and apoptosis ${ }^{[15,16]}$. Cyclindependent kinase inhibitor p21 plays an important role in regulating cell cycle progression, and the apoptosis promoter Bcl-2 Associated X-protein (BAX), which was essential for apoptosis, was also induced by $\mathrm{p} 53^{[17]}$.

In this study, the clinical samples were used to determine the abnormal expression of miRNA-16-5p in GM, and the database was used to analyze and predict the GM-related target genes that miRNA-16-5p may regulate, and to identify the binding sites between GM$5 \mathrm{p}$ and target genes. Then Uppsala87 and Uppsala251 cell models were used for further comprehensive and in-depth analysis of the molecular mechanism of miRNA-16-5p in the treatment of GM, to provide a valuable reference for the same kind of research and development of miRNAs as the target for the treatment of GM.

\section{MATERIALS AND METHODS}

\section{Tissue slice:}

A total of 30 human tissue samples were collected from the pathology department of our hospital, including 10 normal brain samples and 20 GM samples. The samples were immediately frozen in the liquid nitrogen for the following Ribonucleic acid (RNA) analysis, or fixed in the formaldehyde solution for hematoxylineosin staining (H\&E staining). Liquid nitrogen as a part of cryopreservation is widely used for banking cells and tissues which suggests Liquid nitrogen is more advantageous in freezing the tissue samples. The patient understands the study and signs the informed consent form. H\&E staining was carried out according to the above-mentioned scheme.

\section{Cell lines and cell cultures:}

Normal human brain astrocytes (HA) and human brain U87 and U251 cell lines were purchased from China typical Culture Preservation Center (Wuhan). Each cell line was divided into two groups: the experimental group with miR-16-5p agonist (miR-16-5p group) and the negative control group ( $\mathrm{NC}$ group). Each group of cells was cultured in the Dulbecco's Modified Eagle Medium (DMEM) medium supplemented with $10 \%$ fetal bovine serum and 100x penicillin/streptomycin $\left(37^{\circ}, 5 \% \mathrm{CO}_{2}\right)$.

\section{Cell transfection and cloning:}

Cells in the good growth condition $\left(1 \times 10^{5}\right)$ were selected for transfection. The miR-16-5p simulator and negative control simulator were purchased from Gene Pharma Co., Ltd. (Shanghai). According to the operating instructions of the manufacturer, the miR-16-5p simulants and the negative control simulants with the concentration of $62.5 \mathrm{nmol} / 1$ were transferred into the cells by Lipofectamine2000TM (Invitrogen) and cultured for $48 \mathrm{~h}$. The sequence of the simulants were as follows, miR-16-5p stimulants (GCAGGAACUUGUGAGUCUCCU), anti-miR-16$5 p$ stimulants (AGGAGACTCTGTGTTCCTGC). The cells of each group were treated with trypsin and centrifuged to make single cell suspension, and the cells were counted, and the mean value of three times was taken as the result of cell count. The cells were diluted in proportion and inoculated in a 6-well plate according to 200 cells per well. The culture was terminated and stained after 10-12 d of culture until the visible clone ball appeared on the culture plate. Under the microscope, the number of clones of more than 50 cells was counted. Clone formation rate $(\%)=$ number of clones/the inoculated cells*100\%.

\section{MTT detection:}

MTT assay was used to evaluate the cell proliferation and viability. A total of $5 \mathrm{mg}$ MTT reagent (Cell Proliferation Kit I, USA) was dissolved in $1 \mathrm{ml}$ Phosphate-buffered saline (PBS) and filtered by a $0.22 \mu \mathrm{m}$ filter membrane to prepare the reserve solution. The cells were cultured in 96-well plates for $48 \mathrm{~h}$. Then $20 \mu \mathrm{l}$ reserve solution ( $5 \mathrm{mg} / \mathrm{ml}$, Sigma-Aldrich, USA) was added to each well and incubated for $4 \mathrm{~h}$. After the supernatant was removed, $150 \mu$ Dimethyl sulfoxide (DMSO) (Sigma-Aldrich) was added to the plate to dissolve the methanone crystals, and the absorbance of $490 \mathrm{~nm}$ wavelength was measured using an enzyme labeling instrument (Molecular Devices, California, USA).

\section{Determination of transwell migration and invasion:}

Transwell was used to detect the degree of cell migration and invasion. The matrigel coated (invasion assay) or matrigel uncoated (migration assay) membrane was placed in the upper chamber overnight at $37^{\circ}$. After 
cleavage with trypsin, a serum-free medium of about $1 \times 10^{5}$ cells was used to prepare the diluent. The cells were transferred to the upper chamber, and the medium supplemented with $5 \mathrm{mg} / \mathrm{l}$ fibronectin and $10 \%$ Fetal bovine serum (FBS) was added to the lower chamber and cultured for $24 \mathrm{~h}\left(5 \% \mathrm{CO}_{2}, 37^{\circ}\right)$. Cells entering the lower chamber through the membrane were fixed with methanol for about $20 \mathrm{~min}$, followed by staining with crystal violet $(0.1 \%)$ for $10 \mathrm{~min}$. Finally, the cells were examined and counted by a light microscope (Nikon, Japan).

\section{Cell Cycle:}

Apoptosis was detected according to the instructions of FITC Annexin V apoptosis detection kit I. The specific steps were as follows, $1 \times 10^{6}$ cells were collected and re-suspended in $100 \mu \mathrm{l}$ binding buffer. $5 \mu \mathrm{l}$ FITCAnnexin V stain and $5 \mu$ Propidium iodide (PI) stain were added to each test tube, and $400 \mu \mathrm{l}$ binding buffer was added after incubation in the dark for $15 \mathrm{~min}$. Then the apoptosis was evaluated by the flow cytometer within $1 \mathrm{~h}$.

\section{Luciferase reporter gene assays:}

According to the manufacturer's instructions, Phusion Site-Directed Mutagenesis Kit was used to induce the mutagenesis in p53 3'UTR. The wild type and mutant p533'UTR were inserted into the psiCHECK (Promega, Madison, WI, USA), and the recombinant psiCHECK TM-2 vector was cotransfected with miR-16-5p into GM cells. The overexpression of miR-16-5p, miR$16-5 p$ inhibitor or psiCHECK empty vector. Finally, the relative luciferase activity was determined by the luciferase assay reagent II at $48 \mathrm{~h}$ after transfection.

\section{qRT-PCR analyis:}

In this study, RNA was extracted by TRIzol reagent (Invitrogen, USA). According to the instructions of the reagent, the Reverse Transcription Kit (Takara Bio,Inc., Japan) was used to the reverse transcription of the equal portion of RNA into cDNA. The amplification of p53 was carried out on the Biorad Realtime PCR platform. The amplification conditions were as follows, pre-denaturing at $94^{\circ}$ for $2 \mathrm{~min}, 30$ cycles including denaturing at $94^{\circ}$ for $30 \mathrm{sec}$, annealing at $56^{\circ}$ for $30 \mathrm{sec}$, and extending at $72^{\circ}$ for $1 \mathrm{~min}$, and final extending at $72^{\circ}$ for $10 \mathrm{~min}$. GAPDH was used as the endogenous control. Primer sequences were as follows, p53 F: 5'-CCTCAGCATCTTATCCGAGTGG-3', p53 R: 5'-CCTCAGCATCTTATCCGAGTGG-3', BAX F: 5'-CAATTGGCCTTGCTACT-3', BAX R:
5'-TCTTTCGCTGTGAGGTTGTG-3', GAPDH F: 5'-GCA-ACT-AGG-ATG-GTG-TGG-CT-3', GAPDH R: 5'-TCC-CAT-TCC-CCA-GCT-CTC-ATA-3'. The expression level of hsa-mir-127-5p was detected by Taq Man miRNA assay kit. TaqMan MicroRNA Reverse Transcription Kit (Taq Man miRNA RT kit) with miRNA specific RT primers was used to purify and reverse transcribe miRNA. The Reverse transcription polymerase chain reaction (RT-PCR) experiment was carried out on the Step One Plus real-time PCR platform. The reaction mix including $0.2 \mu \mathrm{m}$ Taq Man probe, $2 \mu \mathrm{l}$ RT products, $10 \mu \mathrm{m}$ forward and reverse primers, and $5 \mu$ Taq Man Universal PCR Master Mix.

\section{Western blotting:}

The total protein in the lysate buffer was determined by the radioimmunoprecipitation and separated by SDSPAGE (Bio-Rad, USA) method. Transfer the protein to the polyvinylidene fluoride (PVDF) membrane and blocked it with $5 \%$ skim milk for $1 \mathrm{~h}$. Then the membrane was incubated with the first antibody of $\mathrm{p} 53$ and BAX at $4^{\circ}$ overnight. Next, after incubation with anti-APS IgG-HRP (BOSTER) secondary antibody, the immune complex was revealed by the enhanced chemiluminescence. The relative protein expression was determined by the optical density method using Image J software.

\section{Statistical analysis:}

All the experiments were repeated three times independently. Quantitative data were expressed as mean \pm SD, GraphPad Prism 8.0.1 (GraphPad Software, La Jolla, CA) was used for the comparing and evaluating data between groups. The differences between the two groups were analyzed by t-test. Kruskal-Wallis test was used for comparison among the three groups. $\# \mathrm{p}<0.05$ means the difference was considered significant.

\section{RESULTS AND DISCUSSION}

We analyzed the expression level of the miR-16- $3 p$ in 255 non-cancerous brain GM specimens and 158 different grades of GM tissues from the TCGA public database. The results showed that the expression of miR-16-5p in GM tissues was lower than that in normal tissues (fig. 1A). In addition, the expression level of miR-16-5p in U87 and U251 cell lines was detected. The results showed that the expression of miR-16-5p in the GM cell line was lower than that in a normal brain cell lines (fig. 1B). The expression level of miR-16-5p in the collected tissue slices was analyzed. The results also showed that the miR-16-5p was significantly 
A

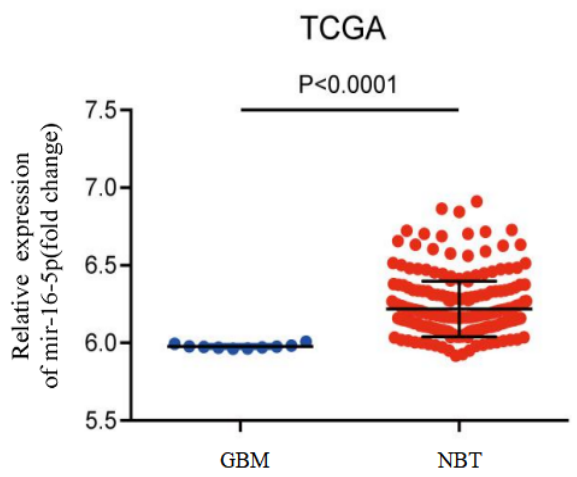

B

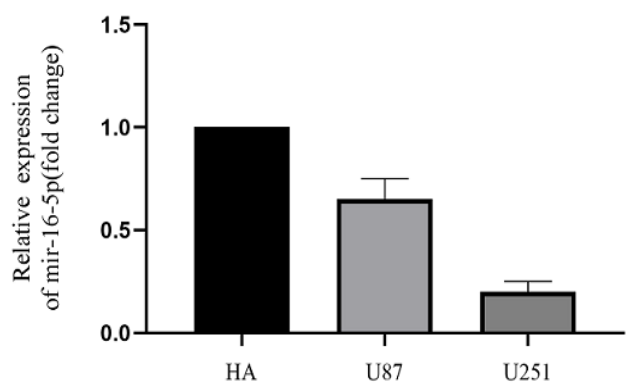

Fig. 1: Low expression of miR-16-5p in GM and GM cells

miR-16-5p expression

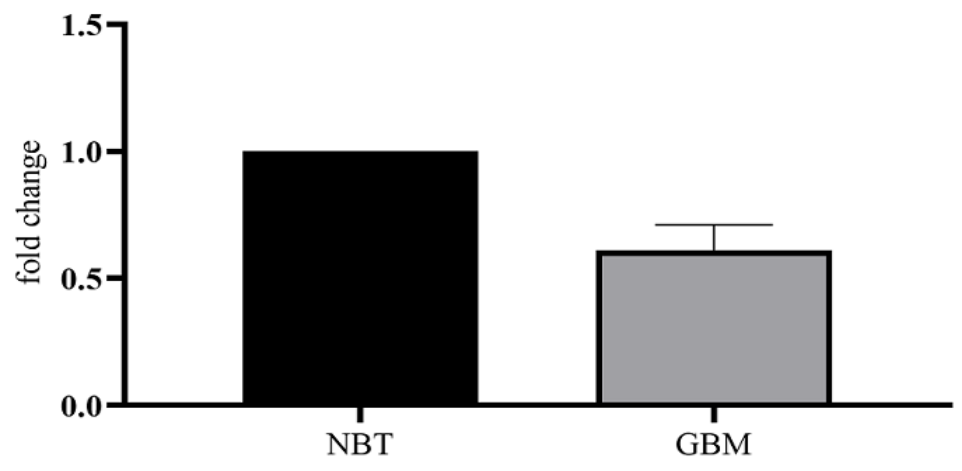

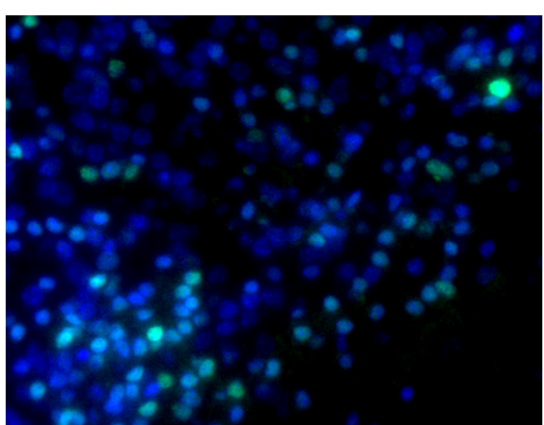

NBT

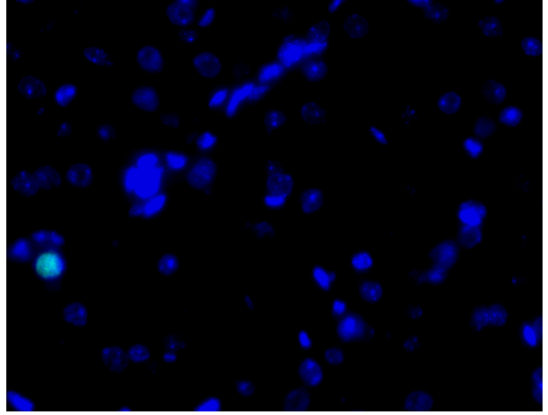

GBM

Fig. 2: Detection of miR-16-5p expression in the normal and GM tissues by qRT-PCR

down-regulated in the GM tissues (fig. 2). These results suggest that the miR-16-5p was lowly expressed in the GM and may play an important role in its occurrence and development.

To study the effect of miR-16-5p on GM cells, U87 and $\mathrm{U} 251$ cells were transfected with the miR-16-5p simulator and miR-16-5p inhibitor simulator. The results showed that the miR-16-5p simulator significantly increased the expression of miR-16-5p (fig. 3), while miR-16-5p inhibitor decreased the expression of miR$16-5 \mathrm{p}$, which indicated that the transfection experiment of miR-16-5p was very successful.
The results of MTT assay showed that compared with the control group (NC group), the proliferation ability of U87 and U251 cells in the miR-16-5p overexpression group decreased significantly, indicating that the overexpression of miR-16-5p inhibited the proliferation of U87 and U251 cells (fig. 4).

The results of Transwell chamber showed that, the number of U87 and U251 cells passing through the chamber decreased significantly when overexpressing miR-16-5p, and the difference was statistically significant $(\mathrm{p}<0.05)$. It was suggested that the overexpression of miR-16-5p can inhibit the invasive 
ability of U87 and U251 cells (fig. 5).

The results of apoptosis analysis showed that the early apoptosis of U87 and U251 cells overexpressing miR$16-5 p$ was significantly increased, and the difference was statistically significant $(\mathrm{p}<0.05)$. At the same time, the results of cell cycle showed that the number of U87 and U251 cells in G1 phase in the miR-16-5p group was significantly higher than that in the control group, while the cells number in $\mathrm{S}$ phase was significantly less than that in the control group, indicating that the overexpression of miR-16-5p could inhibit the cell cycle of U87 and U251 (fig. 6).

The results of the dual-luciferase reporter assay (fig. 7) showed that the activity of the wild-type p53 luciferase decreased significantly after overexpression of miR-16$5 p$, but there was no significant difference in the activity of mutant $\mathrm{p} 53$. These results suggest that the miR-16$5 \mathrm{p}$ can only bind to the wild-type p53 and inhibit the luciferase activity, while the mutant p53 gene cannot. Therefore, $\mathrm{p} 53$ was the target gene of miR-16-5p.

qRT-PCR results showed that the mRNA expression level of p53 and BAX in U87 and U251 cells increased

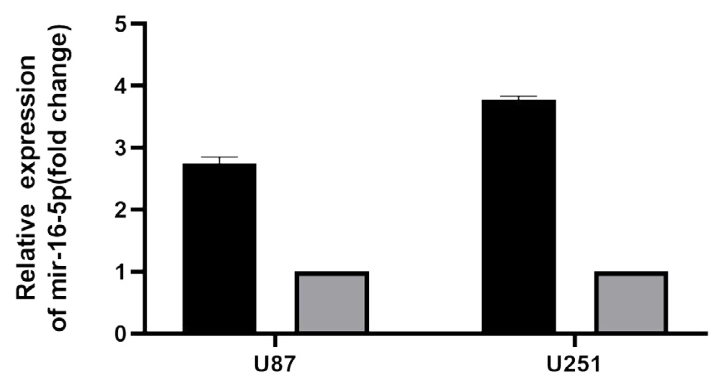

Fig. 3: qRT-PCR analysis of the relative expression level of miR16-5p in $U 87$ and $U 251$ cells after transfection

U87

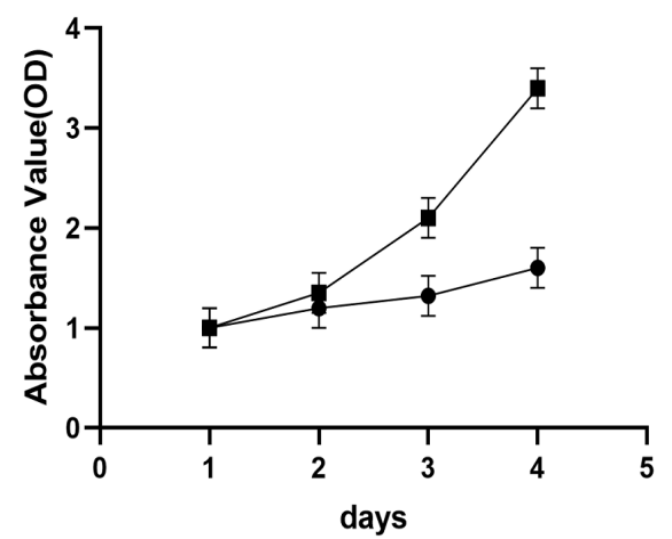

significantly when miR-16-5p was overexpressed. Western blotting also revealed that the miR-16$5 \mathrm{p}$ overexpression could significantly promote the expression of mRNA in U87 and U251 cells, compared with the control group, the difference was statistically significant $(p<0.05)$. These results suggest that the overexpression of miR-16-5p can activate the p53/ BAX signal pathway and inhibit the growth of GM cells (fig. 8).

GM was a malignant tumor in the central nervous system with the highest fatality rate ${ }^{[18]}$. Although the great progress has been made in the clinical diagnosis and targeted therapy in recent $\mathrm{y}$, the $5 \mathrm{y}$ survival rate of patients was still at a low level. The reason for this outcome was not only that this kind of disease occurs in the human brain, but also related to the high invasive ability of the tumor. Therefore, it is necessary to study the biological characteristics and pathological progression mechanism of the tumor at the molecular level, to provide new ideas and methods for the prevention and clinical diagnosis and treatment.

MicroRNAs was a class of small regulatory RNA, that has been shown to activate or inhibit a variety of carcinogenic activities, such as the proliferation, cell cycle, and apoptosis ${ }^{[19]}$. Abnormal expression of miRNA has been observed in various tumors, including brain tumors, such as GM and its invasive GM subtypes ${ }^{[20]}$. Recent studies have shown that the miRNA was involved in the late stage of cancer progression and may be used as an activator or inhibitor of tumorigenesis ${ }^{[21]}$. MiR-16-5p was a member of the miRNA family and plays different roles in different tumors ${ }^{[22-24]}$. This shows that the miR-15-5p was specific in the tumor progression. At present, there were few reports on the role of miR-16-5p in GM and its mechanism, and its

U251

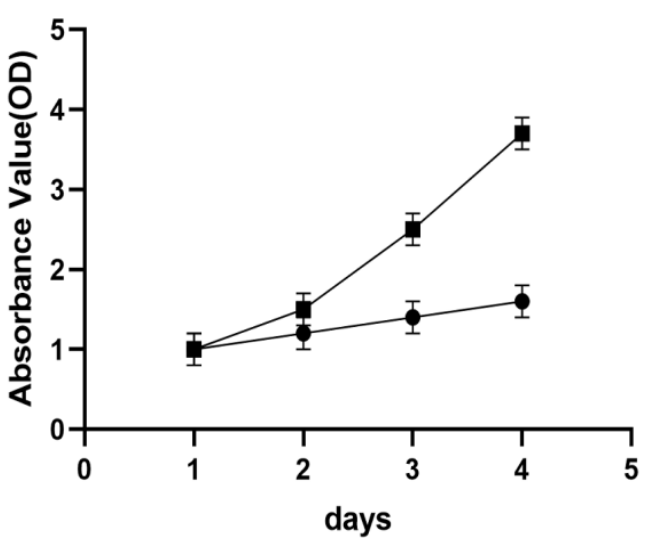

Fig. 4: Proliferation of U87 and U251 cells transfected with anti-miR-ctrl or anti-miR-16-5p 

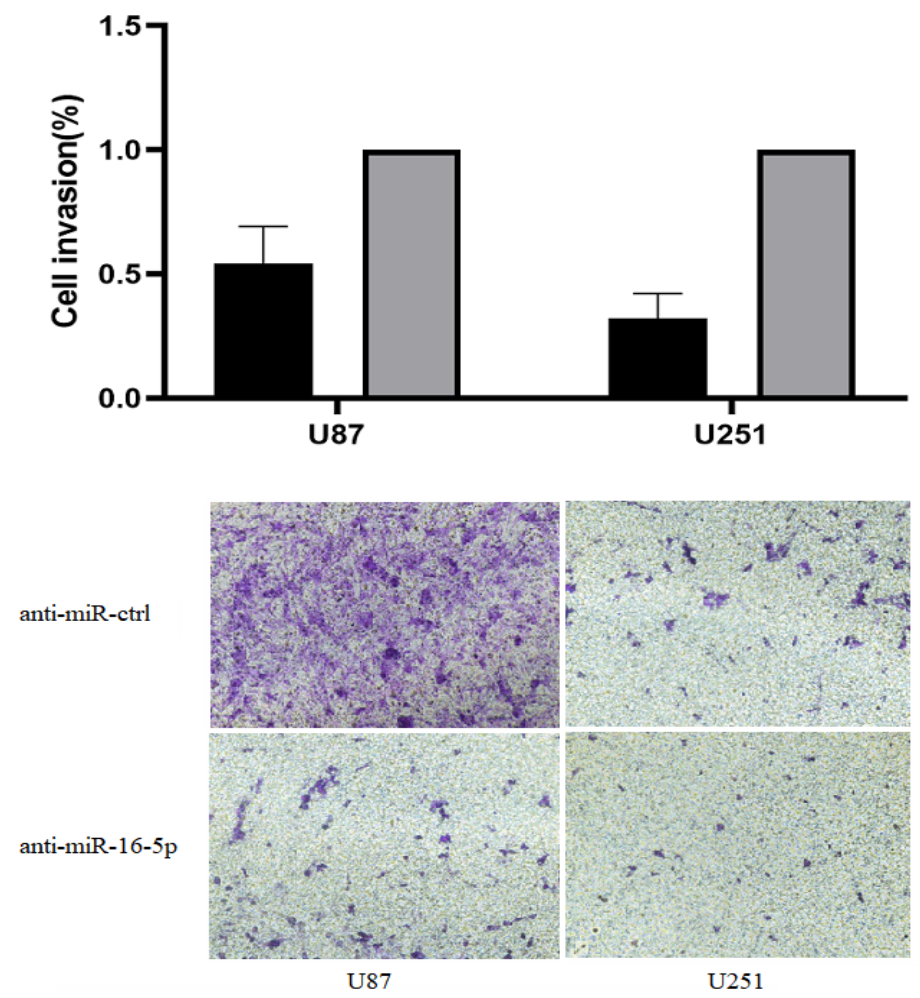

Fig. 5: Detection of the invasive ability of U87 and U251 cells by transwell

U251

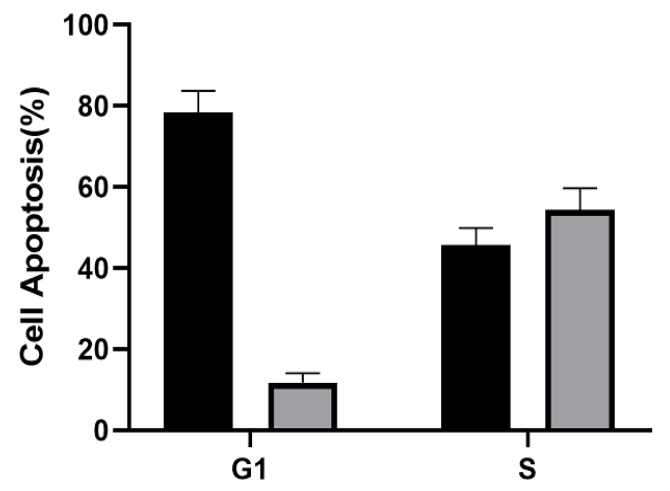

Fig. 6: Apoptosis analysis results of U251 and U87 cells

possible mechanism was not clear. Therefore, in this study, the effect of miR-16-5p on GM and its possible mechanism was studied in vitro cell experiments.

MiR-16-5p has been proved to be a tumor suppressor gene in many tumors. Some studies have found that the miR-16 can target and regulate NF-KB1, MMP-9, and Bcl-2, to inhibit the occurrence and development of $\mathrm{GM}^{[25]}$. Some studies have shown that the miR-16-5p can inhibit the epithelial mesenchymal transition of the transcription factor AP4, thus weakening the invasion and invasion of GM. It was also confirmed that the miR$16-5 p$ could inhibit the growth of GM. Through the analysis of the pathological tissues and normal tissues
U87
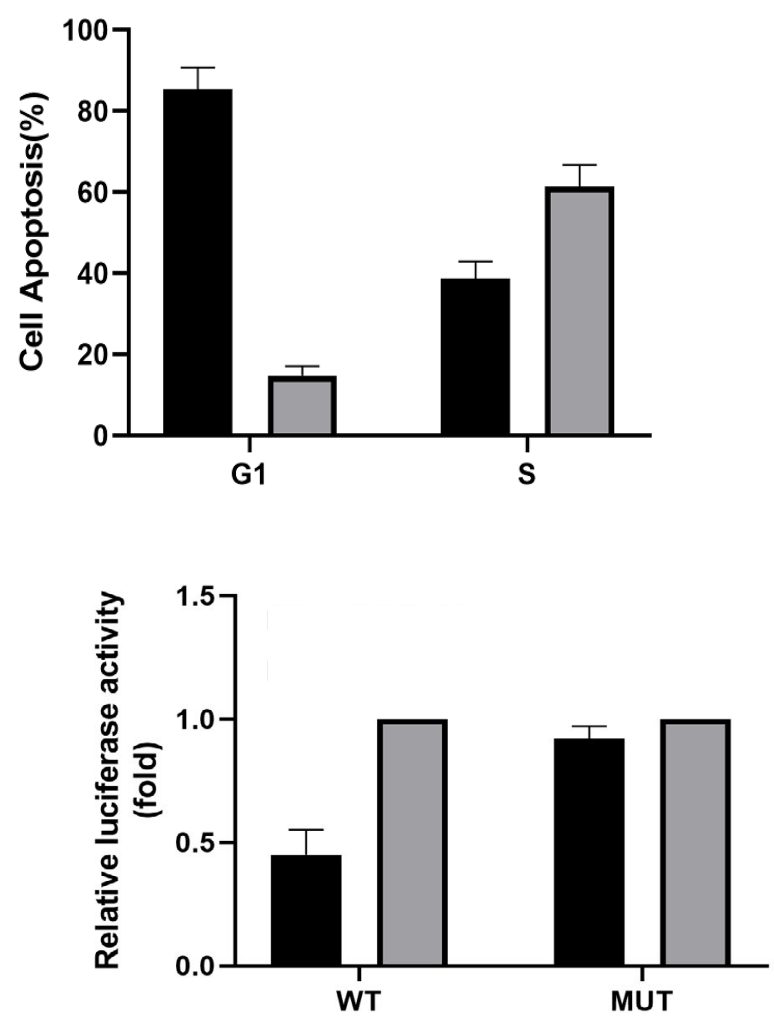

Fig. 7: The results of the dual-luciferase reporter assay

in The Cancer Genome Atlas (TGCA) database, it was found that the expression of the miR-16-5p was low in 
U87

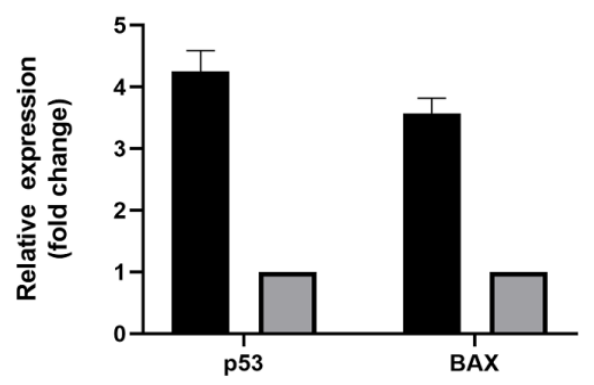

U251

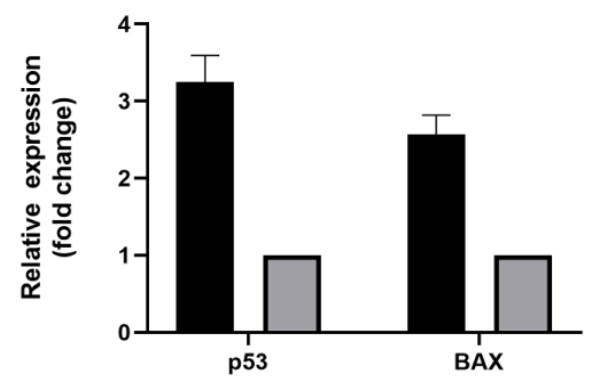

Fig. 8: Detection of relative mRNA expression of $\mathrm{p53}$ and BAX in U87 and U251 cells by qRT-PCR

GM, which was verified in the collected pathological tissues and cell lines respectively. In order to explore the mechanism of miR-16-5p inhibiting the growth of GM, the common GM cell lines U87 and U251 were transfected, and the miR-16-5p overexpression group and control group were set up. Then the characteristics of cell function and biological behavior were tested, including cell proliferation, cell invasion, flow cytometry and apoptosis. The results showed that the overexpression of miR-16-5p could inhibit the cell proliferation and invasion significantly, promote apoptosis and delay cell cycle.

Tumor suppressor gene p53 was a key factor in the development of cancer. When DNA damage occurs, $\mathrm{p} 53^{[26,27]}$ was induced by different upstream signals and then participates in the regulation of cell cycle arrest, DNA repair, and apoptosis-related pathways. In GM, p53 has also been shown to inhibit the occurrence and progression of tumors ${ }^{[28,29]}$. p53 was known to activate a variety of effects, including p21 and BAX, and inhibit the cancer cell growth and tumorigenesis ${ }^{[29,30]}$. Our results showed that the miR-141-3p participates in the tumor inhibition through a variety of mechanisms, including the inhibition of tumor cell growth, promotion of apoptosis, and induction of cell cycle arrest. We have proved that $\mathrm{U} 87$ and $\mathrm{U} 251$ cells were wild-type $\mathrm{p} 53$ cell lines, so the overexpression of miR-16-5p increases the expression of $\mathrm{p} 53$ protein and significantly inhibits the growth of U87 and U251 cells. At the same time, the overexpression of mi-16-5p can increase the level of apoptotic protein BAX in U87 and U251 significantly, which can promote the apoptotic process of GM cells, thus blocking the occurrence and development of GM. Therefore, we believe that the miR-16-5p can activate p53/BAX signal pathway to inhibit the growth of GM effectively.

In summary, all the above data show that the miR-16$5 p$ was a tumor suppressor in the development of GM, and there was a miR-873-5p-p53/BAX regulatory axis in the development of GM, which provides a theoretical basis for the development of alternative strategies for the clinical treatment of GM.

\section{Acknowledgement:}

None

\section{Conflict of Interests:}

The authors declared no conflict of interest.

\section{REFERENCES}

1. Plishka CT, Rotter T, Penz ED, Hansia MR, Fraser SK, Marciniuk DD, et al. Effects of Clinical Pathways for COPD on Patient, Professional, and Systems Outcomes: A Systematic Review. Chest 2019;156:864-77.

2. Tiew PY, San Ko FW, Narayana JK, Poh ME, Xu H, Neo HY, et al. 'High risk' clinical and inflammatory clusters in COPD of Chinese descent. Chest. 2020;158:145-156.

3. Njoku CM, Alqahtani JS, Wimmer BC, Peterson GM, Kinsman L, Hurst JR, et al. Risk factors and associated outcomes of hospital readmission in COPD: A systematic review. Respir Med 2020:105988.

4. Li Y, Zhou J, Rui X, Zhou L, Mo X. PM2. 5 exposure exacerbates allergic rhinitis in mice by increasing DNA methylation in the IFN- $\gamma$ gene promoter in CD4+ T cells via the ERK-DNMT pathway. Toxicol Lett 2019;301:98-107.

5. Hogg JC, Chu FS, Tan WC, Sin DD, Patel SA, Pare PD, et al. Survival after lung volume reduction in chronic obstructive pulmonary disease: insights from small airway pathology. Am J Respir Crit Care Med 2007;176:454-9.

6. Vogelmeier CF, Criner GJ, Martinez FJ, Anzueto A, Barnes PJ, Bourbeau J, et al. Global strategy for the diagnosis, management, and prevention of chronic obstructive lung disease 2017 report. GOLD executive summary. Am J Respir Crit Care Med 2017;195:557-82.

7. Crawford B, Monz B, Hohlfeld J, Roche N, Rubin B, Magnussen $\mathrm{H}$, et al. Development and validation of a cough and sputum assessment questionnaire. Respiratory medicine 2008;102:1545-55.

8. Adeloye D, Chua S, Lee C, Basquill C, Papana A, Theodoratou $\mathrm{E}$, et al. Global and regional estimates of COPD prevalence: Systematic review and meta-analysis. J Glob Health 2015;5:020415.

9. Nielsen LO, Olsen S, Jarbøl DE, Pedersen ML. Spirometry 
in Greenland: a cross-sectional study on patients treated with medication targeting obstructive pulmonary disease. Int $\mathbf{J}$ Circumpolar Health $2016 ; 75: 33258$.

10. Liu MH, Chan MJ, Hsu CW, Weng CH, Yen TH, Huang WH. Association of uremic pruritus in hemodialysis patients with the number of days of high mean 24-hour particulate matter with a diameter of $<2.5 \mu \mathrm{m}$. Ther Clin Risk Manag 2017;13:255-62.

11. Feng S, Duan E, Shi X, Zhang H, Li H, Zhao Y, et al. Hydrogen ameliorates lung injury in a rat model of subacute exposure to concentrated ambient PM2. 5 via Aryl hydrocarbon receptor. Int Immunopharmacol 2019;77:105939.

12. Harrison RM, Laxen D, Moorcroft S, Laxen K. Processes affecting concentrations of fine particulate matter (PM2. 5) in the UK atmosphere. Atmos Environ 2012;46:115-24.

13. Cortez-Lugo M, Riojas-Rodríguez $\mathrm{H}$, Moreno-Macías $\mathrm{H}$, Montes S, Rodríguez-Agudelo Y, Hernández-Bonilla D, et al. Evaluation of the effect of an environmental management program on exposure to manganese in a mining zone in Mexico. Neurotoxicology 2018;64:142-51.

14. Oh SM, Kim HR, Park YJ, Lee SY, Chung KH. Organic extracts of urban air pollution particulate matter (PM2. 5)-induced genotoxicity and oxidative stress in human lung bronchial epithelial cells (BEAS-2B cells). Mutat Res Genet Toxicol Environ Mutagen 2011;723:142-51.

15. Fuentes-Mattei E, Rivera E, Gioda A, Sanchez-Rivera D, Roman-Velazquez FR, Jimenez-Velez BD. Use of human bronchial epithelial cells (BEAS-2B) to study immunological markers resulting from exposure to PM2. 5 organic extract from Puerto Rico. Toxicol Appl Pharmacol 2010;243:381-9.

16. Gualtieri M, Øvrevik J, Holme JA, Perrone MG, Bolzacchini $\mathrm{E}$, Schwarze PE, et al. Differences in cytotoxicity versus proinflammatory potency of different PM fractions in human epithelial lung cells. Toxicol in vitro 2010;24:29-39.

This is an open access article distributed under the terms of the Creative Commons Attribution-NonCommercial-ShareAlike 3.0 License, which allows others to remix, tweak, and build upon the work non-commercially, as long as the author is credited and the new creations are licensed under the identical terms

This article was originally published in a special issue, "Biomedical Research in Clinical and Preclinical Pharmaceutics" Indian J Pharm Sci 2020:82(3)Spl issue7;59-66 